\title{
Output Feedback Stabilization of a Class of MIMO Uncertain Non-affine Nonlinear Systems
}

\author{
Zhenfeng Chen \\ College of Automation \\ Guangdong Polytechnic Normal University \\ No. 293, Zhongshan Avenue, Tianhe District \\ Guangzhou 510665, China
}

\author{
Xuhong Zhang \\ Guangdong Polytechnic \\ Normal University \\ Guangzhou 510665, China
}

\author{
Zhongsheng Wang \\ Guangdong Polytechnic \\ Normal University \\ Guangzhou 510665, China
}

\begin{abstract}
In this paper, robust control design is presented for a general class of uncertain non-affine nonlinear systems. The design employs feedback linearization, coupled with two high-gain observersthe first to estimate the feedback linearization error based on the full state information; the second to estimate the unmeasured states of the system when only the system output is available for feedback. All the signals in the closed loop are guaranteed to be uniform ultimate bounded and the output of the system is proven to converge to a small neighborhood of the origin. The proposed approach not only handles the difficulty in controlling non-affine nonlinear systems, but also simplifies the stability analysis of the closed loop due to its simple control structure.
\end{abstract}

\section{General Terms}

Algorithms, Nonlinear Control Theory

\section{Keywords}

Output feedback control, multi-input/multi-output (MIMO) nonlinear systems, uncertainty

\section{INTRODUCTION}

Recently, controlling nonaffine nonlinear systems becomes an important and challenging topic within the control systems community since, on one hand, many engineering plants are too complex for a mathematical model to describe in the affine form, on the other hand, the main difficulties in controlling nonaffine nonlinear systems come from the decoupling matrix and non-affine appearance of the control input, which makes the extension of control designed for affine to non-affine system generally nontrivial, even if the nonaffine nonlinearity $f$ is known. To handle such a difficulty, several elegant adaptive control schemes were proposed in the literature [1], [7]-[12]. In [7], [8], direct adaptive control methods were proposed via implicit function emulation to achieve the control objectives, which were limited by the lack of the rigorous stability proofs due to the difficulties in analysis. With employment of the Implicit Function Theorem and the Mean Value Theorem, which are not usually associated with neural network (NN) control theory, stable adaptive $\mathrm{NN}$ control and its rigorous stability proof were presented for non-affine nonlinear systems [1]. Based on the idea of feedback linearization techniques, approximated linearizing feedback NN control methods were proposed for a class of non-affine nonlinear systems [9], [10]. In the methods, by adding and subtracting a pseudo-control signal into the plant model, control system design is realized via designing the pseudo-control signal which is required to be invertible with respect to $u$. In [11], [12], the authors suggested to solve the difficulty by adding and subtracting $g u$ directly instead of the pseudo-control signal, where $g$ is a positive constant. This paper is an attempt to express the perspective and to propose a control design method for a general class of uncertain non-affine nonlinear systems. The control design employs feedback linearization and two high-gain observers. The first observer is designed to estimate the feedback linearization error based on the full state information, a robust state feedback controller is developed to compensate the feedback linearization errors. When only the system output is available, then the second observer is employed to estimate the unmeasured states of the system, and a corresponding output feedback controller is proposed. The control performance of the closed loop is guaranteed by suitably choosing the design parameters. By utilizing Implicit Function Theorem and a certain mild condition, the fixed-point problem mentioned above is solved without overly restrictive conditions. The proposed approach not only handles the difficulty in controlling non-affine nonlinear systems, but also simplifies the stability analysis of the closed loop due to its simple control structure, which highlights the simplicity of our proposed control.

The rest of the paper is organized as follows. Section 2 describes the problem formulation. Section 3 presents robust control design based on state feedback and its stability analysis. Section 4 discusses the output feedback control problem. Section 5 illustrates the effectiveness of the proposed controller through examples. Section 6 concludes the paper.

\subsection{Notations and preliminaries}

Throughout the paper, denote $\mathbb{R}$ as the fields of real numbers; $\mathcal{C}$ and $\mathcal{C}^{1}$ respectively as the continuous function and the continuous differentiable function; $|\cdot|$ and $\|\cdot\|$ respectively as the absolute value of a scalar and the norm of a vector/matrix.

LEMMA 1 IMPLICIT FUNCTION THEOREM [13]. Assume that $h: \mathbb{R}^{n} \times \mathbb{R} \rightarrow \mathbb{R}$ is $\mathcal{C}$ for all $(a, b) \in \mathbb{R}^{n} \times \mathbb{R}$ and is 
$\mathcal{C}^{1}$ with respect to $b$. If there exists a constant $c>0$ such that $\partial h(a, b) / \partial b>c, \forall(a, b) \in \mathbb{R}^{n} \times \mathbb{R}$, then, there exists a continuous function $b^{*}=b(a)$ such that $h\left(a, b^{*}\right)=0$.

\section{PROBLEM FORMULATION}

Consider the nonaffine nonlinear systems transformable to the canonical form:

$$
\boldsymbol{y}^{(n)}=\boldsymbol{f}\left(\boldsymbol{y}, \boldsymbol{y}^{(1)}, \cdots, \boldsymbol{y}^{(n-1)}, \boldsymbol{u}\right)
$$

where

u $\in \mathbb{R}^{m}$, the system input;

$\boldsymbol{y} \in \mathbb{R}^{m}$, the system output;

$\boldsymbol{y}^{(i)}$ the $i$ th time derivative of $\boldsymbol{y}$.

$\boldsymbol{f}: \mathbb{R}^{(n+1) \times m} \rightarrow \mathbb{R}^{m}$ is a continuously differentiable function and is completely unknown; $\boldsymbol{\psi}: \boldsymbol{u} \rightarrow \boldsymbol{f}$ is a bijection for every fixed $\boldsymbol{x}=\left[\boldsymbol{y}, \boldsymbol{y}^{(1)}, \ldots, \boldsymbol{y}^{(n-1)}\right] \in \mathbb{R}^{n \times m}$. Unlike most recent results, assume that $f$ is an unknown implicit function with respect to $u$. Without loss of generality, assume that only the output $\boldsymbol{y}$ can be available for feedback and the origin $\boldsymbol{x}=\mathbf{0}$ is an equilibrium point of system (1), i.e., $\boldsymbol{f}(\mathbf{0}, \mathbf{0})=\mathbf{0}$. The difficulties in controlling such systems come from the nonaffine appearance of the control in $\boldsymbol{f}(\boldsymbol{x}, \boldsymbol{u})$, which causes the extension of controls designed for affine to nonaffine systems generally nontrivial. When $\boldsymbol{f}(\boldsymbol{x}, \boldsymbol{u})$ is completely unknown, the controller design for system (1) becomes even more difficult.

Assumption 1. Jacobi Matrix $\partial \boldsymbol{f} / \partial \boldsymbol{u}$ is positive definite and strictly diagonally dominant.

The control objective is to design an output feedback control for system (1) such that the output $y$ is stabilized at the origin, and meanwhile all the signals in the closed-loop system remain uniformly ultimately bounded (UUB).

REMARK 1. Assumption 1 is reasonable because $\partial \mathrm{f} / \partial u$ being nonzero is controllable condition of system (1), which is similarly made in many control schemes [1] 5]. For system (1) with $\partial f / \partial u<$ 0 , it is easily modified in the following analysis.

REMARK 2. Most affine systems (see, e.g., [1]-[3]) can be viewed as the special cases of system (17). The dynamics described in (1) represents many practical nonlinear systems, such as continuous stirred-tank reactor systems [4], vibrating systems [5], and active magnetic bearing systems [6], which are difficult to be exactly described in the affine forms, even though the modeling errors are neglected.

\section{CONTROL SYSTEM DESIGN}

In this section, the presented output feedback control is divided into two parts which are designed in Subsection 3.1 and Subsection 3.2, respectively. And the stability analysis of the entire closed-loop system is presented in Subsection 3.3.

Plus and minus $\boldsymbol{G} \boldsymbol{u}$ to the right-hand side of (1), we obtain

$$
\boldsymbol{y}^{(n)}=\boldsymbol{G u}+\boldsymbol{\Delta}
$$

where $\boldsymbol{\Delta}=\boldsymbol{f}(\boldsymbol{x}, \boldsymbol{u})-\boldsymbol{G} \boldsymbol{u}$ with $\boldsymbol{G}$ being a positive definite matrix. Let the control input be determined as

$$
\boldsymbol{u}=\boldsymbol{G}^{-1}\left(\boldsymbol{u}_{d}+\boldsymbol{u}_{\Delta}\right)
$$

where $\boldsymbol{u}_{d}$ is a dynamic feedback control designed to stabilize system (2) by assuming $\boldsymbol{\Delta}=0 ; \boldsymbol{u}_{\boldsymbol{\Delta}}$ is a filter-based compensator designed to tackle the effect of $\boldsymbol{\Delta}$, i.e., given a small parameter $\varepsilon>0$, find $\boldsymbol{u}_{\boldsymbol{\Delta}}$ such that

$$
\left\|\boldsymbol{u}_{\Delta}+\boldsymbol{\Delta}\right\| \leq \varepsilon, \forall t>0
$$

Due to the bijection $\boldsymbol{\psi}: \boldsymbol{u} \rightarrow \boldsymbol{f}(\boldsymbol{x}, \boldsymbol{u}), \boldsymbol{\Delta}$ depends on $\boldsymbol{u}_{\Delta}$ through $\boldsymbol{f}(\cdot, \boldsymbol{u})$ and $u$, whereas $\boldsymbol{u}_{\Delta}$ has to be designed to tackle $\boldsymbol{\Delta}$, which is the so-called fixed-point solution problem [9]. The following lemma is introduced to solve such a problem.

LEMMA 2. Let

$$
\boldsymbol{h}\left(\boldsymbol{x}, \boldsymbol{u}_{d}, \boldsymbol{u}_{\Delta}\right)=\mathbf{0}
$$

where $\boldsymbol{h}\left(\boldsymbol{x}, \boldsymbol{u}_{d}, \boldsymbol{u}_{\Delta}\right)=\boldsymbol{u}_{\boldsymbol{\Delta}}+\boldsymbol{\Delta}$. Under Assumption 1 , there exists a unique solution $\boldsymbol{u}_{\boldsymbol{\Delta}}^{*}=\boldsymbol{u}_{\boldsymbol{\Delta}}\left(\boldsymbol{x}, \boldsymbol{u}_{d}\right)$ such that $\boldsymbol{h}\left(\boldsymbol{x}, \boldsymbol{u}_{d}, \boldsymbol{u}_{\boldsymbol{\Delta}}^{*}\right)=0$ over the entire input domain of interest.

Proof. From (2), 3), (5) and Assumption 1, we obtain

$$
\boldsymbol{h}\left(\boldsymbol{x}, \boldsymbol{u}_{d}, \boldsymbol{u}_{\boldsymbol{\Delta}}\right)=\boldsymbol{f}(\boldsymbol{x}, \boldsymbol{u})-\boldsymbol{u}_{d}
$$

and

$$
\frac{\partial \boldsymbol{h}\left(\boldsymbol{x}, \boldsymbol{u}_{d}, \boldsymbol{u}_{\boldsymbol{\Delta}}\right)}{\partial \boldsymbol{u}_{\boldsymbol{\Delta}}}=\frac{\partial \boldsymbol{f}(\boldsymbol{x}, \boldsymbol{u})}{\partial \boldsymbol{u}} \frac{\partial \boldsymbol{u}}{\partial \boldsymbol{u}_{\boldsymbol{\Delta}}}
$$

From Lemma 1 there exists a unique solution $u_{\Delta}^{*}=u_{\Delta}\left(x, u_{d}\right)$ such that $h\left(x, u_{d}, u_{\Delta}\right)=0$ over the entire input domain of interest.

REMARK 3. To solve the fixed-point solution problem, some restrictive conditions are required in the previous results, such as a contraction assumption made in [9, 10], and a requirement that $g>\frac{1}{2}(\partial f / \partial u)$ employed in [5]. In this paper, these requirements are removed, which implies that the proposed control scheme can be applied to a wider class of systems.

\subsection{Dynamic feedback controller design}

Under the assumption that $\boldsymbol{\Delta}=\mathbf{0}$, then we can let $\boldsymbol{u}_{\boldsymbol{\Delta}}=\mathbf{0}$ and design $\boldsymbol{u}_{d}$. System (2) reduce as

$$
\boldsymbol{y}^{(n)}=\boldsymbol{u}_{d}
$$

which can be stabilized using classical linear control design, such as the dynamic output feedback control

$$
\begin{aligned}
\dot{\boldsymbol{\xi}} & =\boldsymbol{A}_{d} \boldsymbol{\xi}+\boldsymbol{B}_{d} \boldsymbol{y} \\
\boldsymbol{u}_{d} & =\boldsymbol{C}_{d}^{T} \boldsymbol{\xi}+\boldsymbol{D}_{d} \boldsymbol{y}
\end{aligned}
$$

where $\boldsymbol{\xi} \in \mathbb{R}^{(n-1) \times m}$, and $\boldsymbol{A}_{d}, \boldsymbol{B}_{d}, \boldsymbol{C}_{d}, \boldsymbol{D}_{d}$ are appreciate dimension matrices, which should be chosen such that matrix $\boldsymbol{A}_{s}:=$ $\left[\begin{array}{ccc}\boldsymbol{A}+\boldsymbol{B} \boldsymbol{D}_{d} \boldsymbol{C}^{T} & \boldsymbol{B} \boldsymbol{C}_{d}^{T} \\ \boldsymbol{B}_{d} \boldsymbol{C}^{T} & & \boldsymbol{A}_{d}\end{array}\right]$ is Hurwitz with
$\boldsymbol{A}=\left[\begin{array}{ccccc}\mathbf{0} & \boldsymbol{I} & \mathbf{0} & \cdots & \mathbf{0} \\ \mathbf{0} & \mathbf{0} & \boldsymbol{I} & \cdots & \mathbf{0} \\ \vdots & \vdots & \vdots & \ddots & \vdots \\ \mathbf{0} & \mathbf{0} & \mathbf{0} & \cdots & \boldsymbol{I} \\ \mathbf{0} & \mathbf{0} & \mathbf{0} & \cdots & \mathbf{0}\end{array}\right], B=\left[\begin{array}{c}0 \\ 0 \\ \vdots \\ 0 \\ \boldsymbol{I}\end{array}\right], C=\left[\begin{array}{c}\boldsymbol{I} \\ 0 \\ \vdots \\ 0 \\ 0\end{array}\right]$

\subsection{Compensator design}

Substituting (3) and (8) into (2) yields

$$
\boldsymbol{y}^{(n)}=\boldsymbol{u}_{d}+\boldsymbol{u}_{\boldsymbol{\Delta}}+\boldsymbol{\Delta}
$$


To compensate the effect of $\boldsymbol{\Delta}$, a dynamic high-gain linear filter is constructed by using the available output

$$
\begin{aligned}
\hat{\boldsymbol{y}}_{\Delta}^{(n)} & =-\epsilon^{-1} \boldsymbol{D}_{c}\left(\boldsymbol{y}-\hat{\boldsymbol{y}}_{\Delta}\right)-\boldsymbol{C}_{c}^{T} \boldsymbol{\eta}+\boldsymbol{u}_{d}+\boldsymbol{u}_{\boldsymbol{\Delta}} \\
\epsilon \dot{\boldsymbol{\eta}} & =\boldsymbol{A}_{c} \boldsymbol{\eta}+\epsilon^{-1} \boldsymbol{B}_{c}\left(\boldsymbol{y}-\hat{\boldsymbol{y}}_{\boldsymbol{\Delta}}\right)
\end{aligned}
$$

where $\hat{\boldsymbol{y}}_{\boldsymbol{\Delta}}(\mathbf{0})=\boldsymbol{y}(\mathbf{0})$ and $\boldsymbol{\eta} \in \mathbb{R}^{(n-1) \times m}, \boldsymbol{u}_{\boldsymbol{\Delta}}$ will be designed in (14) and $\epsilon>0$ is a small parameter to be selected later. By introducing the variable

$$
\tilde{\boldsymbol{y}}_{\Delta}:=\epsilon^{-1}\left(\boldsymbol{y}-\hat{\boldsymbol{y}}_{\Delta}\right)
$$

we obtain

$$
\begin{aligned}
\epsilon \tilde{\boldsymbol{y}}_{\Delta}^{(n)} & =\boldsymbol{D}_{c} \tilde{\boldsymbol{y}}_{\Delta}+\boldsymbol{C}_{c}^{T} \boldsymbol{\eta}+\boldsymbol{\Delta} \\
\epsilon \dot{\boldsymbol{\eta}} & =\boldsymbol{A}_{c} \boldsymbol{\eta}+\boldsymbol{B}_{c} \tilde{\boldsymbol{y}}_{\Delta}
\end{aligned}
$$

where $\tilde{\boldsymbol{y}}_{\boldsymbol{\Delta}}(\mathbf{0})=\mathbf{0}$, and $\boldsymbol{A}_{c}, \boldsymbol{B}_{c}, \boldsymbol{C}_{c}, \boldsymbol{D}_{c}$ are appreciate dimension matrices, which should be chosen such that not only matrix $\boldsymbol{A}_{s s}:=$ $\left[\begin{array}{cc}\boldsymbol{A}+\boldsymbol{B} \boldsymbol{D}_{c} \boldsymbol{C}^{T} & \boldsymbol{B} \boldsymbol{C}_{c}^{T} \\ \boldsymbol{B}_{c} \boldsymbol{C}^{T} & \boldsymbol{A}_{c}\end{array}\right]$ is Hurwitz, but also $\tilde{\boldsymbol{y}}_{\boldsymbol{\Delta}}$ converges in a faster time scale than $\boldsymbol{y}$. It is easy to meet this requirement, e.g., setting $\boldsymbol{A}_{c}=\boldsymbol{A}_{d}, \boldsymbol{B}_{c}=\boldsymbol{B}_{d}, \boldsymbol{C}_{c}=\boldsymbol{C}_{d}, \boldsymbol{D}_{c}=\boldsymbol{D}_{d}$ and $\epsilon<1$.

Observing (13a), the compensator is designed as

$$
\boldsymbol{u}_{\boldsymbol{\Delta}}=\boldsymbol{D}_{c} \tilde{\boldsymbol{y}}_{\boldsymbol{\Delta}}+\boldsymbol{C}_{c}^{T} \boldsymbol{\eta}
$$

Combining (13a) and (14), the left-hand side of (4) becomes

$$
\begin{aligned}
& \left|\boldsymbol{u}_{\boldsymbol{\Delta}}+\boldsymbol{\Delta}\right| \\
= & \left|\boldsymbol{D}_{c} \tilde{\boldsymbol{y}}_{\boldsymbol{\Delta}}+\boldsymbol{C}_{c}^{T} \boldsymbol{\eta}+\boldsymbol{\Delta}\right|=\epsilon\left|\tilde{\boldsymbol{y}}_{\Delta}^{(n)}\right|
\end{aligned}
$$

Theory analysis in the next subsection will be shown that appropriate choice of $\epsilon$ can be made such that $\boldsymbol{y}$ is stabilized at the origin and all the signals in the closed loop remain bounded, and thereby (4) holds by further choosing $\epsilon$.

\subsection{Stability analysis}

For clarity, let

$$
\begin{gathered}
\tilde{\boldsymbol{x}}_{\boldsymbol{\Delta}}=\left[\tilde{\boldsymbol{y}}_{\boldsymbol{\Delta}}, \tilde{\boldsymbol{y}}_{\boldsymbol{\Delta}}^{(1)}, \cdots, \tilde{\boldsymbol{y}}_{\boldsymbol{\Delta}}^{(n-1)}\right]^{T} \\
\boldsymbol{X}=\left[\boldsymbol{x}^{T}, \boldsymbol{\xi}^{T}\right]^{T}, \tilde{\boldsymbol{X}}_{\boldsymbol{\Delta}}=\left[\tilde{\boldsymbol{x}}_{\boldsymbol{\Delta}}^{T}, \boldsymbol{\eta}^{T}\right]^{T} \\
\overline{\boldsymbol{B}}=\left[\begin{array}{c}
\boldsymbol{B} \\
\mathbf{0}
\end{array}\right], \boldsymbol{I}_{\epsilon}=\left[\begin{array}{cc}
\boldsymbol{I}_{(n-1) \times(n-1)} & \mathbf{0} \\
\mathbf{0} & \epsilon \boldsymbol{I}_{n \times n}
\end{array}\right]
\end{gathered}
$$

The compact form of system 13 can be written by

$$
\boldsymbol{I}_{\epsilon} \dot{\tilde{\boldsymbol{X}}}_{\boldsymbol{\Delta}}=\boldsymbol{A}_{s s} \tilde{\boldsymbol{X}}_{\boldsymbol{\Delta}}+\overline{\boldsymbol{B}} \boldsymbol{\Delta}
$$

Substituting (3), (8) and (14) into (2), the closed-loop takes the compact form of

$$
\dot{\boldsymbol{X}}=\boldsymbol{A}_{s} \boldsymbol{X}+\overline{\boldsymbol{B}} \overline{\boldsymbol{B}}^{T} \boldsymbol{\Xi}
$$

where $\boldsymbol{\Xi}=\boldsymbol{A}_{s s} \tilde{\boldsymbol{X}}_{\boldsymbol{\Delta}}+\overline{\boldsymbol{B}} \boldsymbol{\Delta}$
The derivative of $\boldsymbol{\Xi}$ is

$$
\begin{aligned}
\dot{\boldsymbol{\Xi}}= & \boldsymbol{A}_{s s} \boldsymbol{I}_{\epsilon}^{-1} \boldsymbol{\Xi}+\bar{B}\left[\frac{\partial \boldsymbol{\Delta}}{\partial \boldsymbol{x}} \dot{\boldsymbol{x}}+\frac{\partial \boldsymbol{\Delta}}{\partial \boldsymbol{u}}\left(\frac{\partial \boldsymbol{u}}{\partial \boldsymbol{u}_{d}} \frac{\partial \boldsymbol{u}_{d}}{\partial \boldsymbol{\xi}} \dot{\boldsymbol{\xi}}\right.\right. \\
& \left.\left.+\frac{\partial \boldsymbol{u}}{\partial \boldsymbol{u}_{d}} \frac{\partial \boldsymbol{u}_{d}}{\partial \boldsymbol{x}} \dot{\boldsymbol{x}}+\frac{\partial \boldsymbol{u}}{\partial \boldsymbol{u}_{\boldsymbol{\Delta}}} \frac{\partial \boldsymbol{u}_{\boldsymbol{\Delta}}}{\partial \tilde{\boldsymbol{x}}_{\boldsymbol{\Delta}}}+\frac{\partial \boldsymbol{u}}{\partial \boldsymbol{u}_{\boldsymbol{\Delta}}} \frac{\partial \boldsymbol{u}_{\boldsymbol{\Delta}}}{\partial \boldsymbol{\eta}}\right)\right] \\
= & \boldsymbol{A}_{s s} \boldsymbol{I}_{\epsilon}^{-1} \boldsymbol{\Xi}+\overline{\boldsymbol{B}}\left(\frac{\partial \boldsymbol{\Delta}}{\partial \boldsymbol{x}}+\frac{\partial \boldsymbol{\Delta}}{\partial \boldsymbol{u}} \boldsymbol{G}^{-1} \boldsymbol{D}_{d} \boldsymbol{C}^{T}\right) \dot{\boldsymbol{x}} \\
& +\frac{\partial \boldsymbol{\Delta}}{\partial \boldsymbol{u}} \boldsymbol{G}^{-1} \overline{\boldsymbol{B}}\left(\boldsymbol{C}_{d}^{T} \dot{\boldsymbol{\xi}}+\boldsymbol{D}_{c} \boldsymbol{C}^{T} \dot{\tilde{\boldsymbol{x}}}_{\boldsymbol{\Delta}}+\boldsymbol{C}_{c}^{T} \dot{\boldsymbol{\eta}}\right) \\
= & \boldsymbol{A}_{s s} \boldsymbol{I}_{\epsilon}^{-1} \boldsymbol{\Xi}+\boldsymbol{\alpha} \dot{\boldsymbol{X}}+\boldsymbol{\beta} \dot{\tilde{\boldsymbol{X}}} \boldsymbol{\Delta} \\
= & \overline{\boldsymbol{A}}_{s s} \boldsymbol{I}_{\epsilon}^{-1} \boldsymbol{\Xi}+\boldsymbol{\alpha}\left(\boldsymbol{A}_{s} \boldsymbol{X}+\overline{\boldsymbol{B}} \overline{\boldsymbol{B}}^{T} \boldsymbol{\Xi}\right)
\end{aligned}
$$

where $\boldsymbol{\alpha}=\overline{\boldsymbol{B}}\left[\frac{\partial \boldsymbol{\Delta}}{\partial \boldsymbol{x}}+\frac{\partial \boldsymbol{\Delta}}{\partial \boldsymbol{u}} \boldsymbol{G}^{-1} \boldsymbol{D}_{d} \boldsymbol{C}^{T}, \frac{\partial \boldsymbol{\Delta}}{\partial \boldsymbol{u}} \boldsymbol{G}^{-1} \boldsymbol{C}_{d}^{T}\right], \boldsymbol{\beta}=$ $\frac{\partial \boldsymbol{\Delta}}{\partial \boldsymbol{u}} \boldsymbol{G}^{-1} \overline{\boldsymbol{B}}\left[\boldsymbol{D}_{c} \boldsymbol{C}^{T}, \boldsymbol{C}_{c}^{T}\right]$ and $\overline{\boldsymbol{A}}_{s s}=\boldsymbol{A}_{s s}+\boldsymbol{\beta}$.

The following theorem shows that the proposed controller stabilizes the origin of system (1) despite the presence of modeling errors $\Delta$.

THEOREM 3. Consider the closed-loop system consisting of nonaffine nonlinear system (1) satisfying Assumption 1 and controller (3), (8) and (14). For bounded initial conditions,

(i) there exists an $\epsilon^{*}>0$ such that for all $0<\epsilon<\epsilon^{*}$, the system output $y$ converges to the origin and all the signals in the closedloop system remain $U U B$;

(ii) given any $\varepsilon>0$, there exists an $\epsilon^{* *}>0$ such that for all $0<\epsilon<\epsilon^{* *}$, the inequality (4) holds.

Proof. (i) The proof is divided into two parts. We first suppose that there exists a compact set $\Omega_{\boldsymbol{x}} \subset \mathbb{R}^{n \times m}$ containing the origin such that $\boldsymbol{x} \in \Omega_{\boldsymbol{x}}, \forall t \geq 0$. Then, we show that this compact set $\Omega_{\boldsymbol{x}}$ do exist for bounded initial conditions.

Consider the Lyapunov function

$$
\boldsymbol{V}=\frac{\boldsymbol{X}^{T} \boldsymbol{P}_{1} \boldsymbol{X}}{2}+\frac{\boldsymbol{\Xi}^{T} \boldsymbol{P}_{2} \boldsymbol{\Xi}}{2}
$$

and the facts that

(a) there exist positive matrices $\boldsymbol{P}_{1}^{T}=\boldsymbol{P}_{1}, \boldsymbol{P}_{2}^{T}=\boldsymbol{P}_{2}$ such that

$$
\boldsymbol{A}_{s}^{T} \boldsymbol{P}_{1}+\boldsymbol{P}_{1} \boldsymbol{A}_{s}=-2 \boldsymbol{I}_{(2 n-1) \times(2 n-1)}
$$

and

$$
\boldsymbol{A}_{s s}^{T} \boldsymbol{P}_{2}+\boldsymbol{P}_{2} \boldsymbol{A}_{s s}=-2 \boldsymbol{I}_{(2 n-1) \times(2 n-1)}
$$

(b) under Assumption 1, the following inequalities hold for all $(\boldsymbol{x}, \boldsymbol{u}) \in \Omega_{\boldsymbol{x}} \times \mathbb{R}^{m}$

$$
\frac{\partial \boldsymbol{f}(\boldsymbol{x}, \boldsymbol{u})}{\partial \boldsymbol{u}} \boldsymbol{G}^{-1}>0
$$

and there exists a dialog positive matrix

$$
\boldsymbol{H}=\left[\begin{array}{ccc}
\boldsymbol{I}_{(n-1) \times(n-1)} & \mathbf{0} & \mathbf{0} \\
\mathbf{0} & \frac{\partial \boldsymbol{f}(\boldsymbol{x}, \boldsymbol{u})}{\partial \boldsymbol{u}} \boldsymbol{G}^{-1} & \mathbf{0} \\
\mathbf{0} & \mathbf{0} & \boldsymbol{I}_{(n-1) \times(n-1)}
\end{array}\right]
$$

such that $\overline{\boldsymbol{A}}_{s s}=\boldsymbol{A}_{s s} \boldsymbol{H}$. 
Then, the derivative of $V$ along the trajectories of the system (17) and $(18)$ yields

$$
\begin{aligned}
\dot{\boldsymbol{V}}= & \boldsymbol{X}^{T} \boldsymbol{P}_{1}\left(\boldsymbol{A}_{s} \boldsymbol{X}+\overline{\boldsymbol{B}} \overline{\boldsymbol{B}}^{T} \boldsymbol{\Xi}\right) \\
& +\boldsymbol{\Xi}^{T} \boldsymbol{P}_{2}\left[\overline{\boldsymbol{A}}_{s s} \boldsymbol{I}_{\epsilon}^{-1} \boldsymbol{\Xi}+\boldsymbol{\alpha}\left(\boldsymbol{A}_{s} \boldsymbol{X}+\overline{\boldsymbol{B}} \overline{\boldsymbol{B}}^{T} \boldsymbol{\Xi}\right)\right] \\
= & -\boldsymbol{X}^{T} \boldsymbol{X}+\boldsymbol{X}^{T}\left(\boldsymbol{A}_{s}^{T} \boldsymbol{\alpha}^{T} \boldsymbol{P}_{2}+\boldsymbol{P}_{1} \overline{\boldsymbol{B}} \overline{\boldsymbol{B}}^{T}\right) \boldsymbol{\Xi} \\
& -\boldsymbol{\Xi}^{T} \boldsymbol{H} \boldsymbol{I}_{\epsilon}^{-1} \boldsymbol{\Xi}+\boldsymbol{\Xi}^{T}\left(\boldsymbol{P}_{2} \boldsymbol{\alpha} \overline{\boldsymbol{B}} \overline{\boldsymbol{B}}^{T}\right) \boldsymbol{\Xi}
\end{aligned}
$$

Further, consider the facts that

(c) since $\Omega_{\boldsymbol{x}}$ is a compact set, and $\boldsymbol{f} \in \mathcal{C}^{1}, \boldsymbol{u} \in \mathcal{C}, \partial \boldsymbol{\Delta} / \partial \boldsymbol{x}$ and $\partial \boldsymbol{\Delta} / \partial \boldsymbol{u}$ are bounded for all $\boldsymbol{x} \in \Omega_{\boldsymbol{x}}$. Therefore, there exist constants $c_{1}>0$ and $c_{2}>0$ such that

$$
\left\|\boldsymbol{A}_{s}^{T} \boldsymbol{\alpha}^{T} \boldsymbol{P}_{2}+\boldsymbol{P}_{1} \overline{\boldsymbol{B}} \overline{\boldsymbol{B}}^{T}\right\| \leq c_{1},\left\|\boldsymbol{P}_{2} \boldsymbol{\alpha} \overline{\boldsymbol{B}} \overline{\boldsymbol{B}}^{T}\right\| \leq c_{2}
$$

(d) the inequality

$$
c_{1}\|\boldsymbol{X}\|\|\boldsymbol{\Xi}\| \leq \frac{1}{2}\|\boldsymbol{X}\|^{2}+\frac{c_{1}^{2}}{2}\|\boldsymbol{\Xi}\|^{2}
$$

(e) $\left\|\boldsymbol{H} \boldsymbol{I}_{\epsilon}^{-1}\right\|=\epsilon^{-1} \varpi$, where $\varpi=\max \left\{\frac{1}{g} \frac{\partial \boldsymbol{f}}{\partial \boldsymbol{u}}, 1\right\}$

Then 22] becomes

$$
\begin{aligned}
\dot{\boldsymbol{V}} & \leq-\|\boldsymbol{X}\|^{2}+c_{1}\|\boldsymbol{X}\|\|\boldsymbol{\Xi}\|-\left(\epsilon^{-1} \varpi-c_{2}\right)\|\boldsymbol{\Xi}\|^{2} \\
& \leq-\frac{1}{2}\|\boldsymbol{X}\|^{2}-\left(\epsilon^{-1} \varpi-\frac{c_{1}^{2}}{2}-c_{2}\right)\|\boldsymbol{\Xi}\|^{2}
\end{aligned}
$$

which means that $\dot{V}<0$ when

$$
\epsilon^{-1} \varpi-\frac{c_{1}^{2}}{2}-c_{2}>0
$$

i.e., $\dot{\boldsymbol{V}}<0, \forall \epsilon \in\left(0, \epsilon^{*}\right)$, where $\epsilon^{*}=\min \left\{\frac{\varpi}{\left(c_{1}^{2} / 2\right)+c_{2}}, 1\right\}$. In addiction, it follows from (19) and 23) that

$$
\dot{\boldsymbol{V}} \leq-c_{3} \boldsymbol{V}
$$

where constant $c_{3}=\min \left\{\frac{1}{\lambda_{\max }\left(P_{1}\right)}, \frac{2 \epsilon^{-1} \varpi-c_{1}^{2}-2 c_{2}}{\lambda_{\max }\left(P_{2}\right)}\right\}$. Solving (25), we obtain that

$$
0 \leq \boldsymbol{V}(t) \leq \boldsymbol{V}(0) e^{-c_{3} t}, \forall t \geq 0
$$

This confirms that for bounded initial conditions and $\epsilon \in\left(0, \epsilon^{*}\right)$, $\boldsymbol{X}$ and $\boldsymbol{\Xi}$ converge to the origin and there do exist a compact $\Omega_{\boldsymbol{x}}$ such that $\boldsymbol{x} \in \Omega_{\boldsymbol{x}}$ for all time.

(ii) Since $\boldsymbol{\Xi}$ converges to the origin, i.e., $\tilde{\boldsymbol{y}}_{\Delta}^{(n)}$ converges to the origin, there exists a constant $c_{4}>0$ such that

$$
\left|\tilde{\boldsymbol{y}}_{\Delta}^{(n)}\right| \leq c_{4}, \forall t>0
$$

Clearly from (4) and (27), given any $\varepsilon>0$, there exists a positive constant $\epsilon^{* *}=\min \left\{\varepsilon / c_{4}, \epsilon^{*}\right\}$ such that for all $0<\epsilon<\epsilon^{* *}$ and for all $\boldsymbol{x}(\mathbf{0}) \in \Omega_{\boldsymbol{x}}$, the controller (3), (8) and (14) satisfies (4).

REMARK 4. The output feedback controller proposed here is easy to implement due to its simply control algorithms with the help of the linear high-gain filter. The control performance of the closedloop system can be achieved by tuning the design parameters. From (24), we obtain that the higher $1 / \epsilon$ is, then the more favorable performance the system exhibits, which will be shown in the simulation results in the next section.
REMARK 5. Compared with the results in [1], [7]-[14], the proposed control has an obvious advantage, i.e., there is no need to construct complex controllers for the non-affine nonlinear systems. Due to its linear control structure, the proposed approach simplifies the stability analysis of the closed loop. From (24), the control performance of the closed-loop system is guaranteed by suitably choosing the parameter $g, l$ and $\epsilon$.

REMARK 6. Since the robust output feedback controller proposed here is simply a state feedback design in combination with a linear high-gain observer without a priori knowledge of the nonlinear systems, it is easy to implement and this is of great significance in engineering practice.

\section{CONCLUSION}

This paper has presented robust control design for a general class of uncertain non-affine nonlinear systems. The design employs feedback linearization, coupled with two high-gain observers - the first to estimate the feedback linearization error based on the full state information; the second to estimate the unmeasured states of the system when only the system output is available for feedback. All the signals in the closed loop are guaranteed to be uniform ultimate bounded and the output of the system is proven to converge to a small neighborhood of the origin. The proposed approach not only handles the difficulty in controlling non-affine nonlinear systems, but also simplifies the stability analysis of the closed loop due to its linear control structure. In the future, investigation on a general class of nonaffine nonlinear systems will be interesting research topics in this field.

\section{ACKNOWLEDGEMENTS}

Supported by National Natural Science Foundation of China (61374003), Foundation for Distinguished Young Talents in Higher Education of Guangdong Province of China (2014KQNCX173), Doctor scientific research of Guangdong Polytechnic Normal University, Project of Guangdong Polytechnic Normal University (14KJY12), Foundation for 1st batch Distinguished Talents in Higher Education of Guangdong Province of China, 2013'.

\section{REFERENCES}

[1] Ge, S. S., Hang, C. C., Zhang, T. 1998. Nonlinear adaptive control using neural networks and its application to CSTR systems. Journal of Process Control. 9, 313-323.

[2] Shiriaev, A. S., Ludvigsen, H., Egeland, O., Fradkov, A. L. 1999. Swinging up of non-affine in control pendulum. In: In Proceedings of American Control Conference, San Diego, California, USA, 4039-4044.

[3] Hsu, C. T., Chen, S. L. 2003. Nonlinear control of a 3-pole active magnetic bearing system. Automatica. 39, 291-298.

[4] Chen, Z. F., Wang, Z. S., Cen, J. 2015. Output feedback stabilization of a class of non-affine nonlinear systems in discrete time. Internatinal Journal of Computer Applications. 119(16), $1-5$.

[5] Park, J. H., Kim, S. H. 2004. Direct adaptive output-feedback fuzzy controller for a nonaffine nonlinear system. IEE Proceedings Control Theory and Applications. 51, 65-72.

[6] Chen, Z. F., Zhang, X. H., Wang, Z. S. 2015. Direct adaptive control for a class of uncertain nonlinear systems. 119(16), 11-15. 
[7] Goh, C. J. 1994. Model reference control of nonlinear systems via implicit funcion emulation. International Journal of Control. 60, 91-115.

[8] Goh, C. J., Lee, T. H. 1994. Direct adaptive control of nonlinear systems via implicit funcion emulation. Control-Theory and Advance Technology. 10 (3), 539-552.

[9] Calise, A. J., Hovakimyan, N., Idan, M. 2001. Adaptive output feedback control of nonlinear systems using neural networks. Automatica. 37, 1201-1211.

[10] Hovakimyan, N., Nardi, F. and Calise, A. J. 2002. A novel error boserver-based adaptive output feedback aproach for control of uncertain systems. IEEE Transactions on Automatic Control. 47 (8), 1310-1314.

[11] Polycarpou M. M., Ioannou P. A. 1996. A robust adaptive nonlinear control design, Automatica. 32(3), 423-427.

[12] Jiang Z. P. and Praly L. 1998. Design of robust adaptive controllers for nonlinear systems with dynamic uncertainties. Automatica. 34, 825-840.

[13] Chen Z. F., Ge S. S., Zhang Y., Li Y. 2014. Adaptive neural control of MIMO nonlinear systems with a block-triangular pure-feedback control structure. IEEE Transactions on Neural Networks and Learning Systems. 25(11), 2017-2029.

[14] Yao B. and Tomizuka M. 1997. Adaptive robust control of SISO nonlinear systems in a semi-strict feedback form. Automatica. 33, 893-900. 